\title{
Maximizing Payload Mass Fractions of Spacecraft for Interplanetary Electric Propulsion Missions
}

\author{
Prashant Patel, ${ }^{*}$ Daniel Scheeres, ${ }^{\dagger}$ and Alec Gallimore ${ }^{\ddagger}$ \\ University of Michigan, Ann Arbor, Michigan 48109-2140
}

\begin{abstract}
Optimization of a spacecraft's interplanetary trajectory and electric propulsion system remains a complex and difficult problem. Simultaneously solving for the optimal trajectory, power level, and exhaust velocity can be difficult and time consuming. If the power system's technology level is unknown, multiple optimizations must be conducted to map out the trade space. Trajectories with constant-power, solar-power, variable-specific-impulse, and constant-specific-impulse low-thrust propulsion systems are analyzed and optimized. The technological variables, power system specific mass, propellant tank coefficient, structural coefficient, and the launch vehicle are integrated into the cost function allowing for maximization of the payload mass fraction. A classical solution is reviewed that allows trade studies to be conducted for constant-power, variable exhaust velocity systems. The analysis is expanded to include bounded-power constant specific impulse systems and solar electric propulsion spacecraft with constant and variable exhaust velocity engines. The cost function and mass fractions are dimensionless to allow for scaling of the spacecraft systems.
\end{abstract}

\section{Nomenclature}

thrust acceleration, $\mathrm{m} / \mathrm{s}^{2}$ launch, $\mathrm{m} / \mathrm{s}^{2}$

launch energy, $\mathrm{km} / \mathrm{s}^{2}$

exhaust velocity, $\mathrm{m} / \mathrm{s}$

launch-vehicle mass fraction

gravitational vector, $\mathrm{m} / \mathrm{s}^{2}$

specific impulse, $s$

payload mass fraction utilized by the spacecraft level at launch

$=$ spacecraft mass, $\mathrm{kg}$

$=$ propellant mass, $\mathrm{kg}$

$=$ propellant mass fraction

$=$ mass flow rate, $\mathrm{kg} / \mathrm{s}$

$=$ jet power, $\mathrm{W}$

$=$ position vector, $\mathrm{m}$

$=$ thrust, $\mathrm{N}$

$=$ time, $\mathrm{s}$

$=$ engine on time, $\mathrm{s}$

$=$ power weighted burn time, $\mathrm{s}$ nominal acceleration spacecraft can deliver at

$=$ characteristic acceleration, $\mathrm{m} / \mathrm{s}^{2}$

optimal exhaust velocity, $\mathrm{m} / \mathrm{s}$

gravity constant defined as $9.8 \mathrm{~m} / \mathrm{s}^{2}$

$=$ quadratic cost function for constant-power variable $I_{\mathrm{sp}}$

$=$ control variable that determines the power fraction

$=$ path and time-dependent function that determines the maximum power fraction as a function of the power

$=$ mass of power system, $\mathrm{kg}$

$=$ power into power processing unit, $\mathrm{W}$

$=$ current-power-to-initial-power utilization ratio

Received 29 April 2005; revision received 16 November 2005; accepted for publication 16 November 2005. Copyright (C) 2006 by Prashant Patel. Published by the American Institute of Aeronautics and Astronautics, Inc., with permission. Copies of this paper may be made for personal or internal use, on condition that the copier pay the $\$ 10.00$ per-copy fee to the Copyright Clearance Center, Inc., 222 Rosewood Drive, Danvers, MA 01923; include the code 0022-4650/06 \$10.00 in correspondence with the CCC.

*Graduate Student, Aerospace Engineering, 1320 Beal Avenue; patelpr@ umich.edu. Student Member AIAA.

${ }^{\dagger}$ Associate Professor, Aerospace Engineering, 1320 Beal Avenue; scheeres@umich.edu. Associate Fellow AIAA.

${ }^{\ddagger}$ Arthur F. Thurnau Professor, Aerospace Engineering, 1320 Beal Avenue; alec.gallimore@umich.edu. Associate Fellow AIAA.

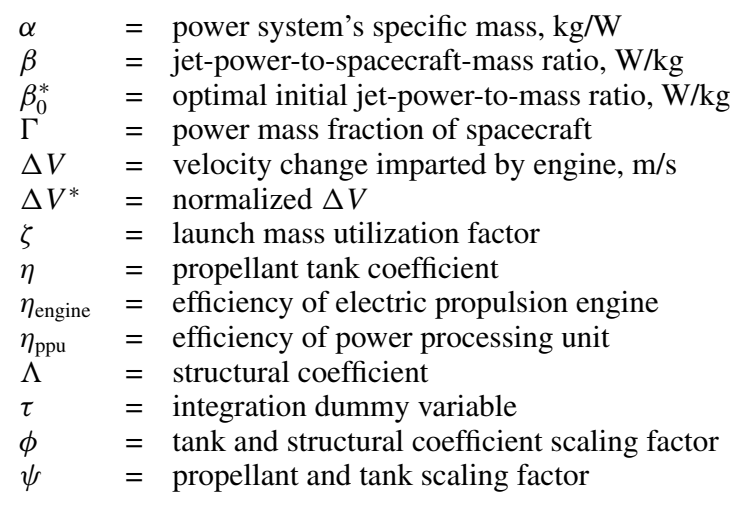

Subscripts and Superscripts

$f \quad=$ final state

$0 \quad=$ initial state

$=$ time derivative

\section{Introduction}

O PTIMIZATION of electric propulsion (EP) spacecraft systems is an active and computationally difficult field of research. In particular, optimization of a combined trajectory, power, and propulsion system is computationally intensive because either a set of coupled two-point boundary-value problems has to be solved or a nonlinear programming problem has to be solved while optimizing over system-level parameters. Furthermore, if the power system's specific mass changes, the problem has to be resolved as the previous solution cannot necessarily be generalized. An alternate and conceptually simpler solution is to map out the system parameter space by computing a trajectory for every combination of $I_{\mathrm{sp}}$, power, and $C_{3}$. The brute force method is simple and robust, but requires a significant amount of computational effort. Also, the brute force method does not provide any insight into the problem of systems optimization.

This paper focuses on maximizing the payload mass fraction for various types of ideal EP engines to facilitate preliminary trade studies. The types of EP systems analyzed are constantpower variable $I_{\mathrm{sp}}$, solar-power variable $I_{\mathrm{sp}}$, constant-power constant $I_{\mathrm{sp}}$, and solar-power constant $I_{\mathrm{sp}}$. The power system's specific mass $\alpha$ is varied to see how it affects the engine parameters, power level, propellant mass, and launch $C_{3}$. The formulation in this paper assumes the efficiency of the engine is constant and 
independent of $I_{\mathrm{sp}}$. The variables that are optimized are $C_{3}, I_{\mathrm{sp}}$, and $P_{j}$.

The most common trajectory optimizers maximize the final mass or minimize the propellant mass for a given mission. ${ }^{1-4}$ Only optimizing the propellant mass can lead to an underutilization of the spacecraft because the power system might be nonoptimal. Previous research has optimized the propulsion and power system,,${ }^{5-7}$ but there has been limited focus on trade studies. This paper addresses all four types of low-thrust systems and nondimensionalizes the cost function. Nondimensionalizing allows for the solution to scale depending on the requirements of the mission. Furthermore, the goal of this paper is to facilitate trade studies instead of optimizing a single design point.

The system parameters referred to in this paper are the spacecraft optimization parameters $C_{3}, I_{\mathrm{sp}}$, and $P_{j}$. The technological parameters are $\alpha, \Lambda$, and $\eta$. The trade studies are conducted over $\alpha$. The technological parameters are the variables that the designer cannot easily or directly control. Generally these variables are determined by the current level of technology. The system parameters are the variables that the spacecraft designer or mission designer has active control over. While trade studies could also be conducted over $\eta$ and $\Lambda$, these two parameters have a weaker effect on the optimization than $\alpha$, and they can be estimated fairly easily.

\section{Engine Models and Motivation}

The large power demands of EP spacecraft make the power system a significant fraction of the vehicle mass. The EP engines affect the motion of the spacecraft through the acceleration term in the equations of motion (1), where $\boldsymbol{G}$ is the gravity vector and $\boldsymbol{a}$ is the thrust acceleration vector:

$$
\ddot{\boldsymbol{r}}=\boldsymbol{G}(\boldsymbol{r}, t)+\boldsymbol{a}(t)
$$

Because power is correlated to thrust, the power system has a direct effect on the dynamics. We define a simple model of an EP engine that relates the jet power to the thrust acceleration term in Eq. (1). The jet power is defined as the power utilized by the engine that results in thrust. The jet power can also be thought of as the power after all efficiency losses have been taken into account. The thrust acceleration is given as the thrust provided by the engine divided by the mass of the spacecraft:

$$
a=T / m
$$

The thrust is related to the jet power of the engine by Eq. (3):

$$
\begin{gathered}
T=2 P_{j} / c \\
P_{j}=\eta_{\mathrm{ppu}} \eta_{\text {engine }} P_{\mathrm{ppu}}
\end{gathered}
$$

More commonly, $I_{\mathrm{sp}}$ instead of $c$ is used, where the definition of $I_{\mathrm{sp}}$ is

$$
I_{\mathrm{sp}}=c / g
$$

$I_{\mathrm{sp}}$ is a measure of how fuel efficient an engine is. At a constant power, as $I_{\mathrm{sp}}$ increases (decreases) thrust and propellant consumption decrease (increase). The mass flow rate of the engine is given as

$$
\dot{m}=-T / c=-2 P_{j} / c^{2}
$$

Substituting these relationships into the acceleration equation, we obtain

$$
a(t)=2 P_{j}(t) / m(t) c(t)
$$

restating the mass

$$
m(t)=m_{0} f\left(C_{3}\right)-\int_{t_{0}}^{t} \dot{m}(\tau) \mathrm{d} \tau=m_{0} f\left(C_{3}\right)-\int_{t_{0}}^{t} \frac{2 P_{j}(\tau)}{c(\tau)^{2}} \mathrm{~d} \tau
$$

we find

$$
a(t)=2 P_{j}(t) / c(t)\left[m_{0} f\left(C_{3}\right)-\int_{t_{0}}^{t} \frac{2 P_{j}(\tau)}{c(\tau)^{2}} \mathrm{~d} t\right]
$$

For an ideal variable $I_{\text {sp }}$ engine an arbitrary acceleration can be delivered simply by increasing or decreasing the $I_{\mathrm{sp}}$ to the appropriate value.

The bounded-power constant $I_{\mathrm{sp}}$ case assumes that the power is limited and that the $I_{\mathrm{sp}}$ is constant. For solar-powered missions, the power varies with time and the distance from the sun and for radioisotope-thermal-generator (RTG)-based missions the power level varies with time. For this reason, a function $k_{2}$, which is a known function of position and time, is introduced, giving

$$
P_{j}(\boldsymbol{r}, t)=k_{2}(\boldsymbol{r}, t) P_{j 0}
$$

Additionally, at any given time the spacecraft might not utilize the available power. For this reason, a second control variable $k_{1}$ is introduced. The variable $k_{1}$ can vary with time and represents the fraction of available power that is utilized by the engine. This gives

$$
P_{j}(\boldsymbol{r}, t)=k_{1}(t) k_{2}(\boldsymbol{r}, t) P_{j 0}
$$

where

$$
0 \leq k_{1} \leq 1
$$

The control is $k_{1}$ while $k_{2}$ is a function of the power source. For convenience we define the following states:

$$
\begin{gathered}
\dot{Z}=k_{1} k_{2} \\
Z(t)=\int_{t_{0}}^{t} k_{1}(\tau) k_{2}(\tau, \boldsymbol{r}) \mathrm{d} \tau
\end{gathered}
$$

This simple EP engine model captures most of the parameters that affect the propellant mass.

\section{Variable $I_{\mathrm{sp}}$}

Variable $I_{\mathrm{sp}}$ implies that the exhaust velocity can be modulated. For an ideal variable $I_{\mathrm{sp}}$ engine any acceleration can be delivered simply by increasing or decreasing the $I_{\mathrm{sp}}$ to the appropriate value. If a variable $I_{\mathrm{sp}}$ engine with a fixed $C_{3}$ is used, only one optimal trajectory has to be found, regardless of the technological factors, as these factors simply scale the solution. If the $C_{3}$ and the trajectory are optimized, the solution will be different for each technological parameter set.

In his classic text Marec ${ }^{8}$ shows that maximizing the payload is equivalent to minimizing the integral of the acceleration squared $J_{2}$ with the assumption of a constant-power, variable $I_{\text {sp }}$, and a constant $C_{3}$. Prussing ${ }^{9}$ shows that an optimal trajectory using $J_{2}$ as the cost function must satisfy a fourth-order differential equation that is solely a function of time and position. Prussing's results make the problem simpler to solve because the adjoint equations do not have to be integrated; however, this reduction only exists if a constantpower, variable $I_{\text {sp }}$ engine is used. The payload mass fraction $J_{1}$ is constructed by taking the final mass and subtracting the power system mass, propellant tank mass, and structural mass, then dividing by the launch vehicle's zero $C_{3}$ mass:

$$
\begin{gathered}
\psi=1+\eta \\
\phi=\Lambda+\eta \\
J_{1}=\frac{m_{\text {payload }}}{m_{0}}=\frac{m_{f}}{m_{0}} \psi-\phi f\left(C_{3}\right)-\alpha \frac{P_{j 0}}{m_{0}} \\
J_{2}=\int_{t_{0}}^{t_{f}} \frac{a(\tau)^{2}}{2} \mathrm{~d} \tau \\
\alpha=\frac{m_{\mathrm{ps}}}{P_{j} \eta_{\mathrm{ppu}} \eta_{\mathrm{engine}}}
\end{gathered}
$$


In Eq. (17) $\alpha$ is the power system's specific mass. We make the usual assumptions that the tank mass scales with the propellant mass, parameterized by the coefficient $\eta$ and that the spacecraft structural mass is a fraction of the launch mass, parameterized by the structural coefficient $\Lambda$. Here $f\left(C_{3}\right)$ is the launch-vehicle's throw mass fraction as a function of $C_{3}$. We require $f(0)=1$, which implies that $m_{0}$ is the throw mass of the launch vehicle for a zero $C_{3}$. The $f\left(C_{3}\right)$ function allows us to maximize the payload mass fraction while taking into account launch-vehicle performance.

For the variable $I_{\mathrm{sp}}$ case we use $\beta$ as a state variable. We differentiate $\beta$ with respect to time and obtain

$$
\begin{gathered}
\beta=P_{j}(t) / m(t) \\
\dot{\beta}=\dot{P}_{j} / m-\dot{m} P_{j} / m^{2}
\end{gathered}
$$

For space propulsion systems the following relationships also apply:

$$
\begin{gathered}
T=m a=-\dot{m} c \\
T=2 P_{j} / c
\end{gathered}
$$

We substitute Eq. (22) and (23) and the definition of $\beta$ into Eq. (21), giving

$$
\dot{\beta}=\left(\dot{P}_{j} / P_{j}\right) \beta+a^{2} / 2
$$

We solve Eq. (24) and find

$$
\beta(t)=\beta_{0} \frac{P_{j}(t)}{P_{j}(0)}+P_{j}(t) \int_{t_{0}}^{t} \frac{a(\tau)^{2}}{2 P_{j}(\tau)} \mathrm{d} \tau
$$

If we assume that $P_{j}$ can be written as $P_{j}=k_{2}(t, r) P_{j 0}$, then Eq. (25) simplifies to

$$
\beta(t)=\frac{k_{2}(t, \boldsymbol{r})}{k_{2}\left(t_{0}, \boldsymbol{r}_{0}\right)}\left[\beta_{0}+\int_{t_{0}}^{t} \frac{a(\tau)^{2} k_{2}\left(t_{0}, \boldsymbol{r}_{0}\right)}{2 k_{2}(\tau, \boldsymbol{r})} \mathrm{d} \tau\right]
$$

We define the integral portion of Eq. (26) as

$$
\begin{gathered}
\Delta \beta=\int_{t_{0}}^{t} \frac{a(\tau)^{2} k_{2}\left(t_{0}, \boldsymbol{r}_{0}\right)}{2 k_{2}(\tau, \boldsymbol{r})} \mathrm{d} \tau \\
\beta_{f}=\frac{k_{2}\left(t_{f}, \boldsymbol{r}_{f}\right)}{k_{2}\left(t_{0}, \boldsymbol{r}_{0}\right)}\left(\beta_{0}+\Delta \beta\right)
\end{gathered}
$$

We substitute Eq. (28) into the $J_{1}$ cost function and obtain

$$
J_{1}=f\left(C_{3}\right)\left[\beta_{0} /\left(\beta_{0}+\Delta \beta\right) \psi-\phi-\alpha \beta_{0}\right]
$$

To maximize $J_{1}$, we must minimize $\Delta \beta$. However, for every value of $\Delta \beta$ there exists a $\beta_{0}^{*}$ that will further maximize $J_{1}$. We take the partial of Eq. (29) with respect to $\beta_{0}$ and set the resulting equation to 0 giving us the value of $\beta_{0}$ that maximizes the original cost function $J_{1}$ as a function of $\Delta \beta$ :

$$
\beta_{0}^{*}=\sqrt{\Delta \beta \psi / \alpha}-\Delta \beta
$$

We substitute the value of $\beta_{0}^{*}$ into the original cost function giving

$$
J_{1}=f\left(C_{3}\right)\left[(\sqrt{\psi}-\sqrt{\alpha \Delta \beta})^{2}-\phi\right]
$$

In its new form we see that the payload mass scales with the initial mass, and for a constant $C_{3}$ minimizing $\Delta \beta$ will maximize $J_{1}$. If the launch vehicle is included, then $J_{1}$ must be minimized instead of $J_{2}$. We apply Marec's assumption of a constant power, $k_{2}=1$, and obtain

$$
\Delta \beta=\int_{t_{0}}^{t_{f}} \frac{a(\tau)^{2}}{2} \mathrm{~d} \tau
$$

which is the same as what Marec derived. Different power systems can be analyzed, by changing the functional forms of $k_{2}$. For example, we can model the solar flux as $\left(r_{0} / r\right)^{2}$, which yields

$$
\Delta \beta=\int_{t_{0}}^{t_{f}} \frac{[r(\tau) a(\tau)]^{2}}{2 r_{0}^{2}} \mathrm{~d} \tau
$$

Once $a(t)$ is known, the exhaust velocity can be computed using the relationship

$$
c(t)=2 \beta(t) / a(t)
$$

Although Marec's derivation is useful for constant-power systems, such as nuclear reactors, it does not apply to solar- or RTGpowered missions. In this section, we have recovered Marec's original result and generalized the result to encompass different power systems.

\section{Bounded Power Constant- $I_{\text {sp }}$}

The use of a constant $I_{\text {sp }}$ fundamentally changes the structure of the problem. Although the state variable $\beta$ can still be used, minimizing $\Delta \beta$ no longer maximizes the payload mass fraction because the acceleration profile cannot be chosen arbitrarily. For this reason, we abandon the preceding formulation and write the payload mass as

$$
\begin{gathered}
\zeta=1-\Lambda \\
\psi=1+\eta \\
J_{1}=f\left(C_{3}\right) \zeta-\psi\left(m_{\text {prop }} / m_{0}\right)-\alpha\left(P_{j} / m_{0}\right)
\end{gathered}
$$

where $\zeta$ represents the mass fraction available at launch. The payload mass fraction is then given by

$$
J_{1}=f\left(C_{3}\right) \zeta-\psi m_{\mathrm{prop}}^{*}-\alpha \frac{1}{2} a_{0} c f\left(C_{3}\right)
$$

We differentiate the propellant mass fraction $m_{\text {prop }}^{*}$ with respect to time and obtain

$$
\dot{m}_{\text {prop }}^{*}=\dot{m} / m_{0}=T / m_{0} c
$$

From the definition of Eq. (14), we reduce Eq. (37) to

$$
\dot{m}_{\text {prop }}^{*}=k_{1} k_{2}\left(T_{0} / m_{0} c\right)=k_{1} k_{2}\left(a_{0} f\left(C_{3}\right) / c\right)=\dot{Z}\left(f\left(C_{3}\right) a_{0} / c\right)
$$

We then integrate, yielding

$$
\begin{gathered}
m_{\text {prop }}^{*}=\frac{a_{0} f\left(C_{3}\right)}{c} Z_{f} \\
Z_{f}=\int_{t_{0}}^{t_{f}} k_{1}(t) k_{2}(\boldsymbol{r}, t) \mathrm{d} t
\end{gathered}
$$

We obtain

$$
J_{1}=f\left(C_{3}\right) \zeta-\psi\left[a_{0} f\left(C_{3}\right) / c\right] Z_{f}-\alpha \frac{1}{2} f\left(C_{3}\right) a_{0} c
$$

by substituting Eq. (39) into Eq. (36). We solve for the optimal exhaust velocity by taking the partial of $J_{1}$ with respect to $c$ and setting the resulting equation to zero:

$$
\begin{gathered}
0=\frac{\partial J_{1}}{\partial c}=f\left(C_{3}\right)\left(\psi Z_{f} \frac{a_{0}}{c^{2}}-\alpha \frac{1}{2} a_{0}\right) \\
c^{*}=\sqrt{\frac{2 \psi Z_{f}}{\alpha}}
\end{gathered}
$$

We reduce Eq. (41) to

$$
J_{1}=f\left(C_{3}\right) \zeta-f\left(C_{3}\right) a_{0} \sqrt{2 \psi \alpha Z_{f}}
$$


by substituting Eq. (43) into the payload mass fraction. We obtain

$$
J_{1}=f\left(C_{3}\right)(\zeta-2 \Gamma)
$$

by replacing $a_{0}$ with the power mass fraction $\Gamma$ :

$$
\Gamma=\frac{1}{2} \alpha a_{0} c
$$

Equation (45) shows that in order to maximize the payload mass fraction we want to maximize $f\left(C_{3}\right)$ and minimize $\Gamma$. $\Gamma$ is the mass fraction of the spacecraft devoted to the power system. Unlike the variable $\beta, \Gamma$ is static and does not change with time. We find

$$
\frac{\alpha P_{j 0}}{m_{0} f\left(C_{3}\right)}=\frac{\psi m_{\text {prop }}}{m_{0} f\left(C_{3}\right)}=\Gamma
$$

by solving for the power mass fraction and propellant mass fraction. We obtain

$$
a(t)=\dot{Z} \sqrt{\left(2 \psi Z_{f} / \alpha\right)}\left\{\Gamma /\left[\psi Z_{f}-\Gamma Z(t)\right]\right\}
$$

by substituting the new variables into Eq. (9). Now we integrate and replace $Z(t)$ with $\Delta V(t)$ and get

$$
a(t)=\dot{Z} \frac{2 \Gamma}{\alpha \Delta V_{f}} \ln \left(\frac{1}{1-\Gamma / \psi}\right)\left(\frac{1}{1-\Gamma / \psi}\right)^{\Delta V(t) / \Delta V_{f}}
$$

where $\Delta V_{f}$ is the total $\Delta V$ the spacecraft delivers for the mission. We define

$$
\begin{gathered}
\Delta V^{*}(t)=\Delta V(t) / \Delta V_{f} \\
a^{*}=1 / \Delta V_{f} \alpha
\end{gathered}
$$

where $a^{*}$ is the characteristic acceleration. We rewrite the acceleration function with the new variables $a^{*}$ and $\Delta V^{*}$ and get

$$
\begin{gathered}
a(t)=k_{1} k_{2} 2 \Gamma a^{*} \ln [\psi /(\psi-\Gamma)][\psi /(\psi-\Gamma)]^{\Delta V^{*}(t)} \\
\Delta \dot{V}^{*}=a(t) a^{*} \alpha=k_{1} k_{2} 2 \Gamma a^{* 2} \alpha \ln [\psi /(\psi-\Gamma)][\psi /(\psi-\Gamma)]^{\Delta V^{*}(t)} \\
c^{*}=\left\{a^{*} \alpha \ln [\psi /(\psi-\Gamma)]\right\}^{-1} \\
J=f\left(C_{3}\right)(\zeta-2 \Gamma)
\end{gathered}
$$

The final transformation removes the condition that $\Delta V\left(t_{f}\right)=\Delta V_{f}$; instead, we have $\Delta V^{*}\left(t_{f}\right)=1$ and $\Delta V^{*}(0)=0$.

\section{A. Constant-Power, Constant $I_{\text {sp }}$}

Constant-power, constant $I_{\text {sp }}$ represents a special case of the bounded-power, constant $I_{\mathrm{sp}}$ solution. The constant-power simplification works for nuclear electric propulsion (NEP) because these systems tend to provide relatively constant power. The simplifying assumption is that $k_{1}=1$ when the thruster is on and $k_{1}=0$ when the thruster is off. We also assume that $k_{2}=1$. We substitute $k_{1}$ and $k_{2}=1$ and obtain

$$
Z_{f}=t_{\text {burnf }}
$$

With the new assumptions, the optimal $I_{\mathrm{sp}}$ is only a function of the burn time. The optimal exhaust velocity ${ }^{10}$ for the constant-power, constant $I_{\text {sp }}$ case is

$$
c^{*}=\sqrt{2 \psi t_{\text {burnf }} / \alpha}
$$

We substitute the burn time into the acceleration function and find

$$
a(t)=\frac{2 \Gamma a^{*} \ln [\psi /(\psi-\Gamma)]}{1-2 t_{\text {burn }}(t) \alpha \Gamma a^{* 2} \ln [\psi /(\psi-\Gamma)]^{2}}
$$

The total burn time is then given as

$$
t_{\text {burn }}=\left\{2 \alpha \psi a^{* 2} \ln [\psi /(\psi-\Gamma)]^{2}\right\}^{-1}
$$

\section{B. Solar-Power, Constant $I_{\mathrm{sp}}$}

With the success of Deep Space One, other missions, such as DAWN ${ }^{\S}$, are taking advantage of solar electric propulsion (SEP). This motivates the next analysis. Like the constant-power, constant $I_{\text {sp }}$ analysis we assume that $k_{1}=1$ or 0 , but now we assume that $k_{2}$ is only a function of the path. For SEP missions we generally find

$$
k_{2}=\left(r_{0} / r\right)^{2}
$$

because the solar flux follows a $1 / r^{2}$ law; more generally, $k_{2}$ can include other factors to take into account solar heating, solar degradation, or minimum power conditions that can preclude the engine from operating after a certain distance from the sun. We obtain

$$
a(t)=\left(r_{0} / r\right)^{2} 2 \Gamma a^{*} \ln [\psi /(\psi-\Gamma)][\psi /(\psi-\Gamma)]^{\Delta V^{*}(t)}
$$

by substituting Eq. (60) into the equations for acceleration. This provides no real reduction or simplifications because the variables are now functions of the path.

\section{Numerical Examples}

Several example trade studies are computed to demonstrate the utility and ease with which trade studies can be conducted. For the NEP-based systems, an Interstellar Probe mission is used. The technical goal of the Interstellar Probe mission is to travel beyond the heliosphere within a reasonable time span. For the SEP-based power systems, an Earth-to-Mars mission is used. The goal of the Mars-based mission is to deliver as much payload as possible to a Mars orbit in 0.5 years. For all of the examples, the phasing is ignored, and the orbits are assumed to be coplaner. The Mars orbit is assumed to be circular at 1.52 astronomical units (AU). The launch vehicle selected for the Earth to Mars transfer is the Atlas V 401. The launch vehicle for the Interstellar Probe mission is the Atlas V 551. The launch vehicle $C_{3}$ curves were parameterized from NASA's Expendable Launch Vehicle Performance Estimation web site. ${ }^{\mathbb{I}}$ The propellant tank coefficient and structural coefficient for all of the examples are set to 0.1 and 0.05 , respectively. Also, the powerprocessing-unit efficiency and the engine efficiency are set to 0.95 and 0.75 , respectively.

The algorithm used to solve the two-point boundary-value problem is a homotopy ${ }^{11}$-based method. First, the algorithm increases the gravitational parameter from 0 to its full value. If SEP is used, the algorithm increases $n$ in Eq. (62) from 0 to 2, after the NEP solution has been found with the gravitational parameter at its full value:

$$
k_{2}=\left(r_{0} / r\right)^{n}
$$

The algorithm is initialized by setting the gravity parameter to 0 and solving the resulting problem, with the boundary conditions that the spacecraft have 0 velocity at the final time for the Mars missions. As the gravitational parameter is increased, the velocity boundary conditions are also increased until they match their correct value. For the Interstellar Probe mission, the final velocity is optimized. After a solution is found with the correct gravitational parameter and power system, the algorithm steps through the $\alpha$ space. The current solution is used as an initial guess for the next value of $\alpha$.

Figures 1-4 are the optimal mass fractions of the various systems. As $\alpha$ increases, the payload mass fraction decreases. Decreasing $\alpha$ always increases the payload mass fraction. The figures quantify the actual benefit. From the figures, we can see that the power system mass fraction does not monotonically increase or decrease with $\alpha$.

For the constant $I_{\mathrm{sp}}$ cases, we need to look at the optimal $I_{\mathrm{sp}}$ to quantify the technology level. For the SEP constant $I_{\text {sp }}$ Earth to Mars mission, Fig. 5 is the optimal $I_{\mathrm{sp}}$ and $C_{3}$ as a function of $\alpha$. We see that the optimal $I_{\mathrm{sp}}$ and $C_{3}$ are in the range of current technology and that as $\alpha$ increases the optimal solution requires a larger $C_{3}$, which indicates the importance of the launch vehicle to the optimal

\footnotetext{
$\S_{\text {Data available online at http://dawn.jpl.nasa.gov/. }}$

ID Data available online at http://elvperf.ksc.nasa.gov/elvMap/index.html.
} 


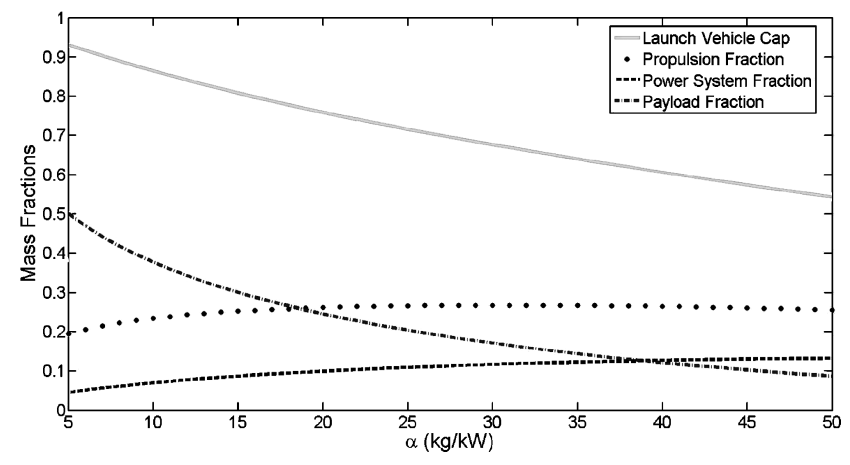

Fig. 1 Mass fraction distribution for the SEP variable $I_{\mathrm{sp}}$ Mars mission.

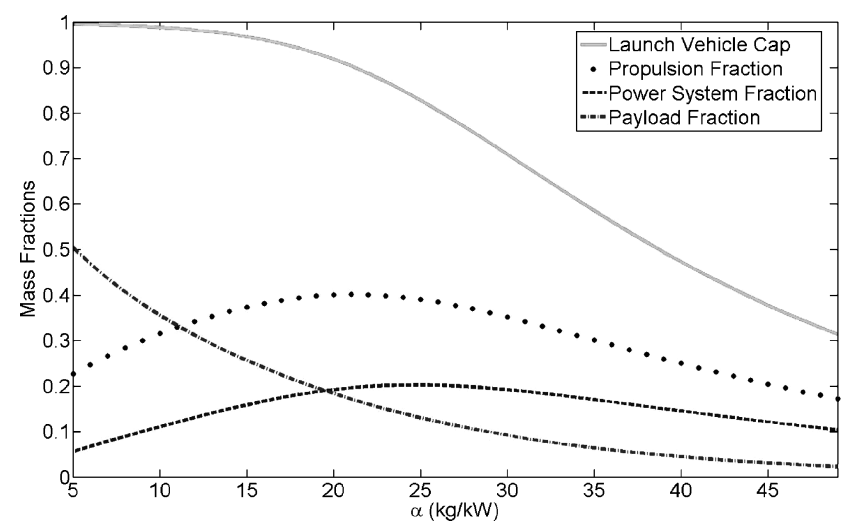

Fig. 2 Mass fraction distribution for the variable $I_{\text {sp }}$ Interstellar Probe mission. The time to $150 \mathrm{AU}$ is constrained to 15 years.

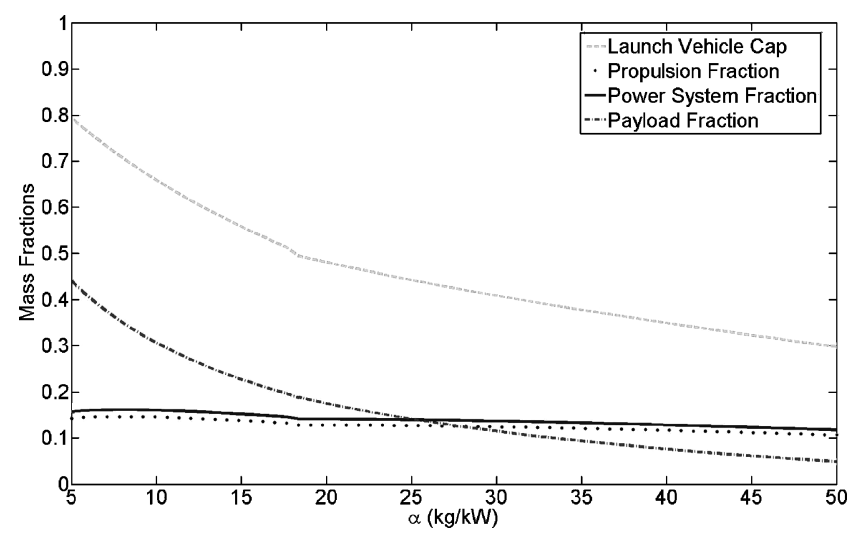

Fig. 3 Mass fractions of the various systems for the Earth-to-Mars SEP constant $I_{\text {sp }}$ case.

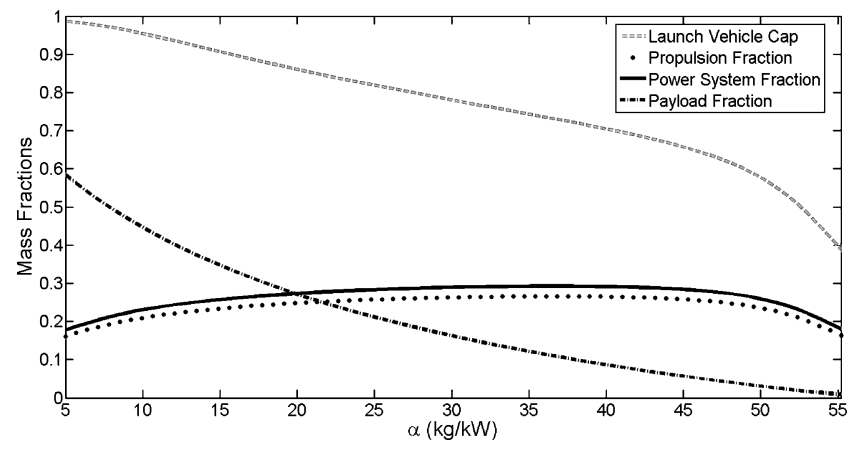

Fig. 4 Mass fractions of the various systems for the Interstellar Probe mission. The program terminated when the payload mass fraction is less than 0.01 .

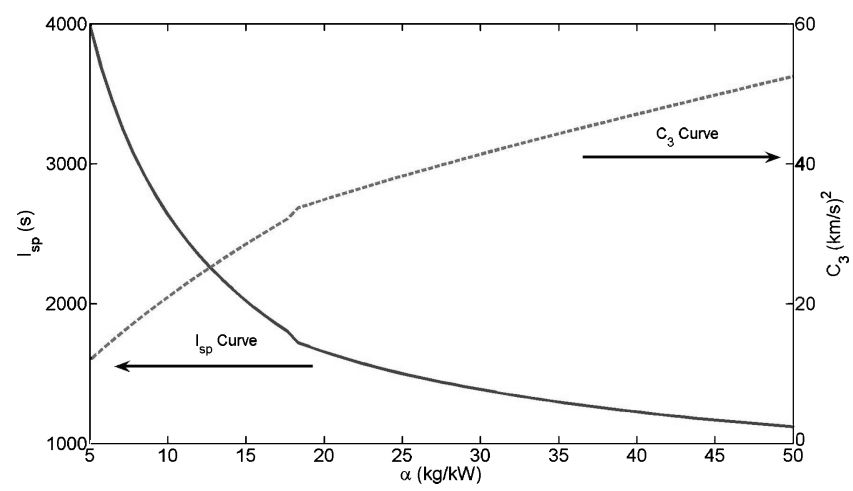

Fig. $5 C_{3}$ and $I_{\mathrm{sp}}$ as a function of $\alpha$ for an Earth-to-Mars transfer in 0.5 years.

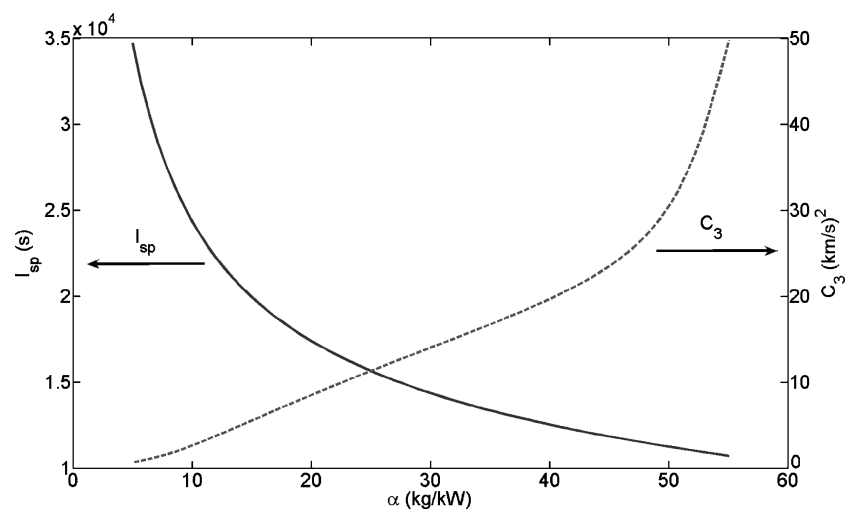

Fig. $6 I_{\mathrm{sp}}$ and $C_{3}$ as a function of $\alpha$ for the constant $I_{\mathrm{sp}}$ Interstellar Probe mission.

solution. Figure 6 is the optimal $I_{\mathrm{sp}}$ and $C_{3}$ as a function of $\alpha$ for the NEP constant $I_{\text {sp }}$ Interstellar Probe mission. Similar to the SEP constant $I_{\text {sp }}$ case, the optimal $I_{\text {sp }}$ decreases, while the optimal $C_{3}$ increases as $\alpha$ increases. However, the range of $I_{\mathrm{sp}}$ are not in the range of current ion engines. The $I_{\mathrm{sp}}$ range would most likely require the use of new propellants.

The total run time for each trade study varies from about $2-5$ mins. The trade study program is written in MATLAB ${ }^{\circledR}$ and is run on a $1.60-\mathrm{GHz}$ Pentium M processor.

\section{Conclusions}

This paper incorporates the propulsion system and the launch vehicle into the cost function transforming the traditional problem of propellant minimization into a true systems optimization problem. The examples serve to show that trade studies can be conducted with relative ease and with little computational effort. Several example trade studies are done to show how the various system elements vary as a function of $\alpha$. Nondimensionalizing the masses makes the procedure general and allows the solutions to scale. We see that variable $I_{\mathrm{sp}}$ and constant $I_{\mathrm{sp}}$ propulsion systems lead to different cost functions. Once a trade study is conducted, explicit technological limits can be found as a function of the mission. This allows a mission designer to know exactly what technological level is required before a mission is feasible.

\section{References}

${ }^{1}$ Yam, C. H., McConaghy, T. T., Chen, K. J., and Longuski, J. M., "Preliminary Design of Nuclear Electric Propulsion Missions to the Outer Planets," AIAA Paper 2004-5393, Aug. 2004.

${ }^{2}$ Whiffen, G., and Sims, J., "Application of the SDC Optimal Control Algorithm to Low-Thrust Escape and Capture Trajectory Optimization," American Astronautical Society, AAS 02-208, Jan. 2002. 
${ }^{3}$ Petropoulos, A., and Longuski, J., "Shape-Based Algorithm for the Automated Design of Low-Thrust, Gravity Assist Trajectories," Journal of Spacecraft and Rockets, Vol. 41, No. 5, 2004, pp. 787-796.

${ }^{4}$ Rayman, M. D., and Williams, S. N., "Design of the First Interplanetary Solar Electric Propulsion Mission," Journal of Spacecraft and Rockets, Vol. 39, No. 4, 2002, pp. 589-595.

${ }^{5}$ Kluever, C. A., and Chang, K.-R., "Electric-Propulsion Spacecraft Optimization for Lunar Missions," Journal of Spacecraft and Rockets, Vol. 33, No. 2, 1996, pp. 235-239.

${ }^{6}$ Lorenzo Casalino, G. C., and Pastrone, D., "Optimal Low-Thrust Escape Trajectories Using Gravity Assist," Journal of Guidance, Control, and Dynamics, Vol. 22, No. 5, 1999, pp. 637-642.

${ }^{7}$ Casalino, L., and Colasurdo, G., "Optimization of Variable-SpecificImpulse Interplanetary Trajectories," Journal of Guidance, Control, and Dynamics, Vol. 27, No. 4, 2004, pp. 678-684.
${ }^{8}$ Marec, J. P., Optimal Space Trajectories, Elsevier Scientific, New York, 1979, pp. 15-17.

${ }^{9}$ Prussing, J. E., "Equation for Optimal Power-Limited Spacecraft Trajectories," Journal of Guidance, Control, and Dynamics, Vol. 16, No. 2, 1993, pp. 391-393.

${ }^{10}$ Casalino, L., and Colasurdo, G., "Trade-off Between Payload and TripTime for EP Interplanetary Trajectories," AIAA Paper 2004-3805, July 2004.

${ }^{11}$ Minter, C., and Fuller-Rowell, T., "A Robust Algorithm for Solving Unconstrained Two-Point Boundary Value Problems," American Astronautical Society, AAS 05-129, Jan. 2005.

J. Korte Associate Editor 